\title{
Transatlantica
}

Revue d'études américaines. American Studies Journal

\section{Pinocchio et Barbie prennent le Transatlantique}

A propos des expositions d'Olivier Rebufa et de Jim Dine

Marie Cordié Lévy

\section{CpenEdition}

Journals

Édition électronique

URL : http://journals.openedition.org/transatlantica/1862

DOI : $10.4000 /$ transatlantica. 1862

ISSN : $1765-2766$

Éditeur

AFEA

Référence électronique

Marie Cordié Lévy, "Pinocchio et Barbie prennent le Transatlantique », Transatlantica [En ligne], 2 |

2007, mis en ligne le 29 janvier 2008, consulté le 29 avril 2021. URL : http://journals.openedition.org/ transatlantica/1862 ; DOI : https://doi.org/10.4000/transatlantica.1862

Ce document a été généré automatiquement le 29 avril 2021.

\section{(c) (i)}

Transatlantica - Revue d'études américaines est mis à disposition selon les termes de la licence Creative Commons Attribution - Pas d'Utilisation Commerciale - Pas de Modification 4.0 International. 


\title{
Pinocchio et Barbie prennent le Transatlantique
}

\author{
A propos des expositions d'Olivier Rebufa et de Jim Dine
}

Marie Cordié Lévy

- Olivier Rebufa : Once Upon a Time, du 25 avril au 16 juin 2007 à la galerie Beaudoin Lebon, 38 rue Sainte Croix de la Bretonnerie, 75004 Paris

- Jim Dine : Aldo et moi, du 24 avril au 17 juin à la crypte de la BNF, site Richelieu

- L'Odyssée de Jim Dine, Estampes 1985-2006, du 16 mars au 11 juin au Musée des Beaux Arts de Caen

- Jim Dine : Pinocchio. Edition limitée à 35 exemplaires, composée de 41 planches lithographiées exposée à la galerie Artcurial, Hotel Dassault, 7 Rond Point des Champs Elysées, 75008 Paris

Deux icônes du monde de l'enfance, la poupée Barbie de la firme Mattel et le Pinocchio de Collodi viennent de traverser l'Atlantique main dans la main et d'arriver à Paris, l'une pour envahir l'espace de la galerie Baudoin Lebon à travers l'œuvre photographique d'Olivier Rebufa, l'autre pour se trouver perché au-dessus des livres de la docte librairie Artcurial.

2 C'est Barbie qu'a choisi Olivier Rebufa pour revisiter les grandes scènes de l'histoire de l'art. La première étape de sa démarche consiste à passer de sa taille d'homme à celle des 29 centimètres de sa poupée plastique lilliputienne. Pour métamorphoser l'homme de chair et d'os à l'homme de papier, Olivier Rebufa se photographie sous tous les angles afin de passer de l'autre côté du miroir :

J'achète ensuite les jouets, les poupées et ustensiles nécessaires à la réalisation de la scène. Tout peut servir, légo, gadgets, bibelots, objets magnétiques pour frigos, etc... Les poupées sont démantelées, découpées, soumises à des transformations pour obtenir la pose désirée. Je réalise alors mon autoportrait [...]. Ce tirage, où je suis cadré à la dimension des poupées me sert à réaliser après découpage ma propre marionnette que j'insère, après l'avoir renforcée au dos avec du fil de fer, dans le décor dimension jouet. La dernière étape consiste à photographier cette mise-enscène.

(Olivier Rebufa, 2000) 
3 Une fois réduit, tel John Malkovich dans le film Dans la peau de John Malkovich, il peut alors se laisser envahir par ses fantasmes les plus délirants et la Barbie l'y invite : sans père ni mère, la poupée de plastique fortement sexuée, grâce à ses mensurations de mannequin, devient une chose qui se prête à tous les jeux interdits qu'il lui propose.

4 On peut ici regretter l'accrochage saturé de la galerie Baudoin Lebon. Un choix plus sélectif, qui aurait suivi par exemple son odyssée mythologique intitulée Saison des hommes dieux, aurait sans doute été plus satisfaisant. Parce que les cartels manquent, le visiteur est contraint à un jeu de devinette. Étant donné le lieu d'ancrage de l'artiste, Marseille et la Méditerranée, le visiteur subodore déjà, à la vision de son corps poilu, tour à tour ailé, cornu, ou aux pattes de satyre, que quelques mythes grecs risquent d'apparaître. En effet, identifiant Psyché et Cupidon, Léda et le cygne, Pan et Syrinx, Pégase et Athéna, Pâris, Thésée et le Minotaure, Orphée et Eurydice, Narcisse et Echo, Zeus et Gaïa, Diane et Actéon, Ariane et Dionysos (photos toutes prises en 2001), le visiteur découvre un espace de liberté où l'artiste jongle avec de multiples facettes de lui-même, comme un enfant joue à changer d'identité: " on dirait que toi tu serais... et moi je serais... "

Ce jeu, qu'Olivier Rebufa explore dans une atmosphère de kamasoutra débridé, devient une véritable mise en abyme, quand, suivant le parcours psychique de tous les grands amateurs d'art, il réinvente les tableaux du Louvre avec une méticulosité loufoque : on reconnaît ainsi une Piéta et une Annonciation, la Cène de Léonard de Vinci, la Chute d'Icare de Breughel, Adam et Eve chassés du Paradis terrestre de Masaccio, le Cyclope de Goya et des tableaux d'inspiration orientaliste comme Le Fumeur d'opium.

6 Self-made man éclectique, Olivier Rebufa s'attaque aussi aux icônes des temps modernes tels que Mastroianni, Mickey Rourke ou Humphrey Bogart, réinterprète les classiques hollywoodiens comme Casablanca ou African Queen ou les chefs d'œuvre du cinéma italien, comme la Dolce Vita ou Huit et demi.

7 Mais de cette série d'autoportraits multiples coincée au milieu d'un amas de figurines « cosmétiques » selon le mot de Paul Ardenne dans le brillant article qu'il lui a consacré ( Olivier Rebufa, Universal Hero »11), naît parfois un sentiment de malaise mélancolique, comme si le héros, à jamais enfermé dans ce dialogue muet, se débattait, tel Gulliver pris au piège des Lilliputiens, sans jamais réussir à reprendre figure humaine.

8 Le second jouet à portée fortement autobiographique est le Pinocchio utilisé par Jim Dine. Dans une magnifique série exposée à Caen et chez Artcurial, l'artiste réinvestit l'icône enfantine avec la maîtrise et la force d'un des plus grands lithographes de ce siècle. Présentées dans une boîte en planches xylographiée du même bois que son jouet fétiche, les lithographies sont toutes à l'échelle américaine : grandes, belles et d'une lisibilité parfaite. L'incrustation de parties du texte de Collodi dans l'image a été mise au point par l'imprimeur Woolworth avec qui Jim Dine a travaillé pour cette série. Loin d'être un lettrage sage et poli qui respecte l'image, celui-ci la bouscule, l'obstrue ou la cache, à la façon des grands comics américains ou d'une manière qui rappelle l'omniprésence du texte dans le paysage américain (on pense par exemple aux affiches et pancartes photographiées par Walker Evans). Les lambeaux de textes apparaissent dans différentes couleurs ou polices de caractère suivant l'humeur de l'histoire. Les mots peuvent envelopper Pinocchio comme un petit manteau bleu (voir la lithographie où Gepetto vient de faire de nouveau pieds à Pinocchio, Geppetto Makes Pinocchio a New Pair of Feet, and Sells His Coat to Buy Him), établir un commentaire distant sur l'action (en rouge dans Pinocchio is in Danger), plagier en bleu la démarche fourbe des méchants (The 
Fox and the Cat) ou rester collés à ses habits quand les assassins le pendent (The Assassins Pursue). Retrouvant la force des sons qui frappent l'imaginaire enfantin, ils ânonnent sur l'image, et, comme les enfants qui répètent les phrases des contes, entraînent à une totale empathie avec l'émotion véhiculée par le texte.

Dans un entretien avec Caroline Joubert, conservatrice du musée des Beaux Arts de Caen, Jim Dine explique ainsi son intérêt pour ce texte sur lequel il travaille depuis 1999:

Ce qui m'a conduit à lui est que je suis allé voir le film de Walt Disney quand j'étais petit et ce fut une expérience traumatisante. J'étais un petit garçon menteur, et dans l'histoire, comme dans le livre de Collodi, les méchants petits garçons sont punis. Ce petit garçon reste pour moi un personnage extrêmement touchant et je veux le célébrer, prendre soin de lui².

10 Si Jim Dine retourne à cette histoire, c'est parce qu'il peut, selon ses propres termes, "penser à l'envers»: cette expression, utilisée à propos de l'estampe, s'applique parfaitement à sa démarche. Comme au jeu de l'oie, il retourne à la case départ, revient à rebours de ce qu'il est devenu et retourne au présent via l'art. De ce voyage intérieur vers ce qui l'anime, l'artiste veut laisser des traces sur l'image, petits cailloux blancs que sont les frissons d'émotion le long de la route de la création. Se comparant tour à tour à Pinocchio et à Gepetto, il explique ainsi sa démarche à Caroline Joubert :

C'est clairement une métaphore de la création artistique. On donne à Gepetto un bâton qui parle. Il le taille pour en faire une figure de garçon. Le pantin de bois traverse un " enfer » pour devenir une personne réelle. C'est ainsi que «l'art» se fait ».

11 Jim Dine, qualifié tour à tour d'artiste pop ou print junkie, étiquettes par trop catégorisantes, revendique pour lui-même le terme d'hokusaï, c'est-à-dire «vieux garçon fou d'estampes ». Mais n'est-il que cela? Puisant son inspiration chez les plus grands lithographes tels que Rembrandt, Munch, Giorgio Morandi, l'alchimiste-enfant en quête d'une forme émotionnelle ontologique se place aux côtés de Picasso ou de David Hockney, artistes qui ont également travaillé avec A.Crommelynck. En choisissant Pinocchio, il troque le jouet-pistolet de Lichtenstein pour un jouet moins brutal, plus évocateur, riche des possibles d'une véritable quête, avec ses erreurs et ses errances (l'école buissonnière, les mauvaises rencontres) et au bout du compte, la promesse d'une récompense, sous la forme d'une belle lithographie.

À propos de l'exposition «Aldo et Moi »

12 Dans la crypte de la Bibliothèque Nationale, Jim Dine réinvestit les icônes les plus célèbres de l'art occidental pour leur donner une signification universelle. Par un jeu de déhanchement, la Vénus de Milo rejoint celle de Botticelli. La lithographie intitulée The Foam, c'est-à-dire l'écume, est imprimée à l'envers. Par ce geste de retournement magique, Jim Dine veut-il montrer l'envers de la beauté, et/ou nous dire que l'artiste seul sait en percevoir le secret?

13 Parmi les différents motifs représentés, on note cet enveloppant peignoir fermé (The Robe), protecteur de sa nudité, devenu la signature, pour ainsi dire la marque de fabrique de l'artiste. Autre motif récurrent dans son œuvre, l'icône du cœur se craquèle comme un morceau d'écorce terrestre (voir la lithographie exposée à Caen) ou se dédouble en cœur brûlant des amants éternels, Roméo et Juliette, dormant à jamais dans la crypte (ici celle de la BNF). Intitulée Ex-voto, cette magnifique lithographie de deux cœurs rouges placée au centre même de l'exposition, avec d'un côté les 
lithographies représentant sa femme et de l'autre, la série des vanités et des Vénus, pourrait être par le titre même un hommage au poème de Baudelaire: A une madone, ex-voto dans le goût espagnol ${ }^{3}$.

En parallèle, d'autres lithographies tout aussi spectaculaires remplissent l'espace, comme ses outils de graveur (New French Tools, Tools and Dreams), les quatre continents (Four continents) ou les coquillages (Lost shells). Les outils peuvent à leur tour être vus comme une autocitation (Five Feet of Colorful Tools, 1962) ou symboliser le pragmatisme américain du «Just do it » quand ils sont représentés alignés sur le skyline new yorkais. Quant aux deux coquillages, signes premiers de vie sur terre, ils évoquent les photos de Weston faites dans le désert de Point Lobos.

Avec ces bois palimpsestes, l'exposition «Aldo et moi» marque le retour de l'artiste à la source de sa création et peut se lire comme un hymne universel et secret à l'amour éternel qui flotte bouillonnant, impétueux, inextinguible, au dessus de ces deux villes, Paris et New York.

7 juin 2007, Paris

rois questions à Marie-Cecile Miessner, conservateur en chef, chargée de l'estampe contemporaine à la Bibliothèque Nationale et commissaire de l'exposition "Aldo et moi » accrochée dans la crypte.

1/ Comment le projet de cet événement autour de l'œuvre de Jim Dine a-t-il vu le jour entre la Bibliothèque Nationale, le musée des Beaux-Arts de Caen et la librairie Artcurial?

Pourquoi Jim Dine a-t-il choisi Paris pour cette exposition?

Jim Dine a fait une donation à la Bibliothèque Nationale avec la perspective de faire une exposition alors que le projet d'une exposition à Caen existait déjà. Il y a donc eu deux expositions Jim Dine presqu'en même temps.

A Caen ont été exposées les lithographies de grand format faites chez Pace à partir des bois. Le musée des Beaux Arts de Caen expose en outre l'album Pinocchio édité par Steidl, imprimé par Woolworth à Paris, qui comprend 34 lithographies. Cet album a déjà été présenté en Belgique, au Passage des Princes, en novembre 2006, à la New York Public Library, en décembre 2006. Il est actuellement exposé à Caen et chez Artcurial.

21 Ici à Paris, Jim Dine a voulu remercier son ami et imprimeur, Aldo Crommelynck, qui a réalisé pour lui une centaine de planches. C'est pour rendre hommage à cet homme qui lui a fait aimer Paris que Jim Dine a fait ce don à la France.

2/En quoi son travail sur Pinocchio est-il révélateur de sa recherche actuelle?

23 Jim Dine est un artiste polyvalent. Il a fait le catalogue de la galerie Pace, mais a aussi produit des sculptures de Pinocchio, tout comme il avait fait une sculpture monumentale de la Vénus de Milo précédemment pour le Guggenheim de Bilbao.

3/ Quelle technique Jim Dine utilise-t-il ? Quels sont les thèmes majeurs abordés dans cette exposition?

Ces grandes estampes sont rehaussées à la peinture acrylique, ou à l'aquarelle, comme la Vénus de Milo dans The Foam. Jim Dine a rehaussé la totalité des trente estampes exposées ici. Ce sont de véritables œuvres murales.

On retrouve ici les thèmes récurrents qui rappellent sa traversée du pop' art comme les outils, les vêtements. Mais ici ils n'ont plus cette résonance et deviennent des thèmes du quotidien, de sa vie personnelle, comme le peignoir ou les French Tools. La Vénus de 
Milo (The Foam) qui sort de l'onde est un hommage aux antiquités du Louvre. Les coquillages sont ceux qu'il allait chercher à vélo chez les taxidermistes, quand il travaillait avec Crommelynck. On peut les lire comme des paysages qui montrent l'objet dans sa dimension profonde, sa force de vie. Le magnifique diptyque rouge intitulé Ex Voto, représente deux cœurs anatomiques, deux cœurs de chair. Ils rappellent le cœur exposé à Caen, craquelé comme une planète.

Les outils sont à lire comme une écriture avec des majuscules : si on regarde les deux plaques les représentant, on voit une plaque neutre où les outils figurent simplement et une autre intitulée Tools and Dreams où ils symbolisent le New York skyline, avec les deux cœurs qui flottent au-dessus. Le fond frotté est bouillonnant, en action, à l'image de ce grand artiste.

\section{NOTES}

1. Paul Ardenne, «Olivier Rebufa, Universal Hero », (Paris : Baudoin Lebon. 2006).

2. L'odyssée de Jim Dine (Musée des Beaux Arts de Caen : Steidt, 2006).

3. Je veux bâtir pour toi, Madone, ma maîtresse, Un autel souterrain au fond de ma détresse, Et creuser dans le coin le plus noir de mon cœur, Loin du désir mondain et du regard moqueur, Une niche d'azur et d'or tout émaillée, Où tu te dresseras, statue émerveillée.

INDEX

Thèmes : Trans'Arts 